\title{
Article \\ Efficient Covering of Thin Convex Domains Using Congruent Discs
}

\author{
Shai Gul ${ }^{1, *}$ and Reuven Cohen ${ }^{2}$
}

1 Department of Applied Mathematics, Holon Institute of Technology, Holon 5810201, Israel

2 Department of Mathematics, Bar-Ilan University, Ramat-Gan 5290002, Israel; reuven@math.biu.ac.il

* Correspondence: shaigo@hit.ac.il

Citation: Gul, S.; Cohen, R. Efficient Covering of Thin Convex Domains Using Congruent Discs. Mathematics 2021, 9, 3056. https://doi.org/ $10.3390 /$ math 9233056

Academic Editors: Árpád Bûrmen, Tadej Tuma and Alexander Felshtyn

Received: 1 October 2021

Accepted: 23 November 2021

Published: 28 November 2021

Publisher's Note: MDPI stays neutral with regard to jurisdictional claims in published maps and institutional affiliations.

Copyright: (c) 2021 by the authors. Licensee MDPI, Basel, Switzerland. This article is an open access article distributed under the terms and conditions of the Creative Commons Attribution (CC BY) license (https:// creativecommons.org/licenses/by/ $4.0 /)$.

\begin{abstract}
We present efficient strategies for covering classes of thin domains in the plane using unit discs. We start with efficient covering of narrow domains using a single row of covering discs. We then move to efficient covering of general rectangles by discs centered the lattice points of an irregular hexagonal lattice. This optimization uses a lattice that leads to a covering using a small number of discs. We compare the bounds on the covering using the presented strategies to the bounds obtained from the standard honeycomb covering, which is asymptotically optimal for fat domains, and show the improvement for thin domains.
\end{abstract}

Keywords: covering; thin domains; optimal placement

\section{Introduction}

In discrete geometry, finding an efficient covering of a given domain using unit discs is a well known problem, with various applications such as facility location and cellular network design. The covering problem is strongly related to the well-studied packing problem [1-4].

It has been known for a long time that the optimal covering of the entire two dimensional plane is obtained using the honeycomb (regular hexagonal) lattice, where the plane is tiled by regular hexagons, and the center of each hexagon is used as the center of the inscribing disc. The fact that this is indeed the best obtainable covering was proven about a century ago by Thue and Toth $[5,6]$.

In [7], an upper bound for covering any convex domain with area $A$ and perimeter $L$ has been given. It has been shown that by the properties of the hexagonal regular lattice, the number of unit discs needed to cover a convex domain is at most

$$
\left\lfloor\frac{2}{3 \sqrt{3}} A+\frac{2}{\pi \sqrt{3}} L+1\right\rfloor .
$$

This result is obtained using a probabilistic method by a random (location and orientation-wise) placement of the convex domain on the hexagonal lattice. It has been shown that the expectation of the number of unit hexagons in the hexagonal regular lattice that hit the domain is given by the area of the Minkowski sum of the regular hexagon and the convex domain. The expectation value of this area can be calculated using mixed area formulas, and this expectation leads to this upper bound on the number of required discs in the most efficient covering. In [8], another estimation using geometric probability has been presented, in this case, for pairs of hyperplanes intersecting a convex body.

In [9], an algorithm for finding the exact location and orientation of the given convex domain relative to the lattice is presented, such that the number of unit discs in the cover is minimized (for a given lattice). In particular, the cardinality of the cover given by the algorithm is bounded from above by the bound given in Equation (1). 
Also in [9], a lower bound for the number of unit discs necessary to cover a convex domain has been given. If $n_{\text {opt }}$ is the optimal number of unit discs that cover a convex domain, then $n_{\mathrm{opt}} \geq \max \left\{\frac{2 A}{3 \sqrt{3}}, \frac{L}{4}\right\}-C$, for some absolute constant $C>0$.

For fat domains, it follows that the honeycomb-based covering is asymptotically optimal. However, for thin domains, there are known cases in which the honeycomb covering is far from optimal. In this manuscript, we present some case studies in which the honeycomb covering is suboptimal and present improved coverings that are based on deformations of the hexagonal lattice.

In [10], a thinnest covering has been described for a rectangle and a unit square using up to 7 congruent discs.

In [11], an algorithm for finding the minimal covering of convex polygons by two minimum diameter congruent discs is presented.

In [12], an efficient algorithm for covering for line segments in the plane by unit discs is presented.

In this paper, we present some results on covering several families of domains efficiently. The first part will consider covering a general convex thin domain, which can be either a polygon or a domain with curved boundary. The second part considers rectangles. However, many of the results presented in the second part apply also to general polygons, as well as non-polygonal domains. Most of the presented results also apply to thin nonconvex domains. For a general discussion of covering convex and non-convex domains see [9].

\section{Preliminaries}

Definition 1. The Minkowski sum of any two sets $A, B \subset \mathbb{R}^{2}$ is defined to be $A+B:=\{x+y$ : $x \in A, y \in B\}$. For $s \geq 0$, the Minkowski dilation by factor $s$ is defined to be $s A=\{s x: x \in A\}$.

Definition 2. For every $\theta \in[0,2 \pi)$, the support function of a domain $\Omega$, denoted $h(\theta)$, is a function that maps every $\theta$ to the supremum over all $p$ such that $L(p, \theta) \cap \Omega \neq \varnothing$, where $L(p, \theta)$ is the line $\{(x, y) \mid \cos (\theta) x+\sin (\theta) y=p\}$. The width of $\Omega$ is $w(\theta)=h(\theta)+h(\theta+\pi)$.

We state some standard theorems from integral geometry. We refer the reader to [7] or other standard textbooks in the field for full proofs.

Theorem 1 (Cauchy's Formula [7]). Let $\Omega$ be a bounded convex domain, then the circumference of $\Omega$ is given by

$$
L(\partial \Omega)=\int_{0}^{2 \pi} h(\theta) d \theta=\int_{0}^{\pi} w(\theta) d \theta
$$

where $h$ is the support function of $\Omega$ and $w$ is $\Omega$ 's width.

Theorem 2 ([7]). Suppose $\Omega$ is a compact, convex domain with a continuous boundary. Then, the area of $\Omega$ is

$$
A(\Omega)=\frac{1}{2} \int_{0}^{2 \pi}\left(h^{2}-h^{\prime 2}\right) d \theta,
$$

where the support's derivative $h^{\prime}$ may be a distribution.

\section{Row Covering}

Consider a long and narrow domain. The expression in Equation (1) is given an upper bound on the number of discs needed to cover it. However, this expression is determined by two variables $A$ (area) and $L$ (perimeter). If the domain is narrow enough, it is expected that a covering can be obtained that depends almost solely on $L$ since the main restriction on the covering is that it must cover all the length of the perimeter. Thus, it desired to give an improved upper bound as well as a proper definition of "narrow enough". 
Notation 1. Consider a convex domain $\Omega$ with area $A$ and perimeter $L$. Let $d$ denote the diameter of $\Omega$ and $h$ its height, which will be calculated as the width in a direction perpendicular to the diameter (in case the diameter is determined by several chords, we choose one arbitrarily).

Our assumption that such an improvement indeed exists is based on the following simple observation. Take a very narrow $(h \rightarrow 0)$ convex domain $\Omega$. Then, the number of unit discs that are needed to cover $\Omega$ tends to

$$
\frac{d}{2} \approx \frac{L / 2}{2}=\frac{L}{4} .
$$

Comparing (3) to (1) gives us the justification that, indeed, such an improvement exists.

Proposition 1. For a convex domain $\Omega$ having diameter $d$ and height $h, \Omega$ is bounded by $a$ rectangle with sides $d$ and $h$.

Proof. The diameter is the longest chord in the domain. Taking the endpoints of the diameter, no point in the domain is at a distance more than $d$ from those points. Thus, the domain is inscribed in the intersection of two circles of radius $d$ centered at the diametrically opposing points, and therefore also between the tangents to those circles at the diametrically opposing point. Since the height is determined by the maximum distance of two parallel lines to $d$, which intersect $\Omega$, this result follows.

Definition 3. A parallel row covering of a convex domain $\Omega$ is a cover composed of $n$ unit discs in a row, which is parallel to the diameter (or any one of the diameters, if more than one exists), as in Figure 1.

It should be noted that there are row coverings that are not parallel row coverings. For instance, row covering a rectangle can be done naturally along its longer side, and not its diameter (which is any one of the two diagonals).

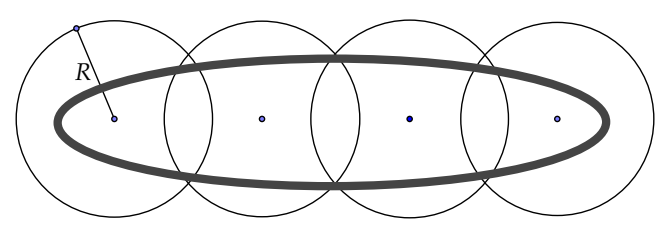

Figure 1. Parallel row covering of an ellipse.

Lemma 1. Given a convex domain $\Omega$ and having diameter $d$ and a height $h$. If $h=2-\varepsilon, \varepsilon>0$, then there exists a parallel row covering to $\Omega$ with at most

$$
\left[\frac{d}{2 \sqrt{1-(1-\varepsilon / 2)^{2}}}\right]+1
$$

unit discs.

Proof. Since every convex domain is bounded by a rectangle with length $d$ and height $h$, it is sufficient to cover the rectangle. W.1.o.g, we will locate the rectangle with the diameter parallel to the $x$-axis with the leftmost point touching the $y$-axis and with $h$ cut in half by the $x$-axis (i.e., the uppermost point and the lowermost point will be at the same distance of $h / 2$ from the $x$-axis). 
Let $G(x, y, \lambda)=(x-\lambda)^{2}+y^{2}-1$. Define the family of unit circles, parametrized by $\lambda$, whose centers lie on the $x$-axis to be the set of solutions of the equations

$$
C_{\lambda}=\{(x, y) \mid G(x, y, \lambda)=0\},
$$

and the appropriate unit discs bounded by these circle as

$$
D_{\lambda}=\{(x, y) \mid G(x, y, \lambda) \leq 0\} .
$$

Every two nearby circles intersect at two points if and only if the equation $C_{\lambda} \cap C_{\lambda+h}$ consists of two points. If the distance between these two intersection points is greater than $h$, then the set of unit discs $D_{\lambda+n h}$ for some appropriate set $n \in A \subset \mathbb{Z}$ constitutes a covering of the domain.

Denote the distance between the centers of two nearby circles by $2 a$. The distance from the $x$-axis to the intersection point between two nearby circles is $\frac{h}{2}$ (since the covering by circles is symmetric respective to the $x$-axis). This leads to $1=\sqrt{(h / 2)^{2}+a^{2}}$. Since $h=2-\varepsilon$, the number of unit circles is

$$
n:=\left[\frac{d}{2 a}\right]+1=\left[\frac{d}{2 \sqrt{1-(1-\varepsilon / 2)^{2}}}\right]+1 .
$$

We note that the center of the first unit circle will be located at a distance $\lambda=$ $\sqrt{1-\left(1-\frac{\varepsilon}{2}\right)^{2}}$ from the leftmost edge of the rectangle, which determines the locations of the $(n-1)$ unit discs that complete the covering.

Theorem 3. Given a convex domain $\Omega$

1. If there exists a parallel row covering for $\Omega$, then $\frac{A}{L}<2$.

2. If $\frac{A}{L}<\frac{1}{\pi}$, then $\Omega$ admits a parallel row covering.

Proof. The following bounds hold for every convex domain $\Omega$ with area $A$ and perimeter $L$ :

$$
\sqrt{d^{2}+h^{2}} \leq L \leq \pi \cdot d
$$

The lower bound in Equation (4) results by constructing two triangles with bases on the diameter and the uppermost and lowermost points (respectively) as a third vertex, as in Figure 2. By convexity, the perimeter $\Omega$ is at least the perimeter of these triangles, which obtains its maximum of $\sqrt{d^{2}+h^{2}}$ when both triangles are isosceles and congruent. The upper bound in Equation (4) is obtained by replacing the width function in Theorem 1 by the diameter $d$.

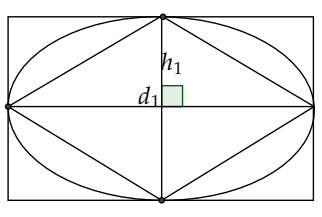

$(I)$

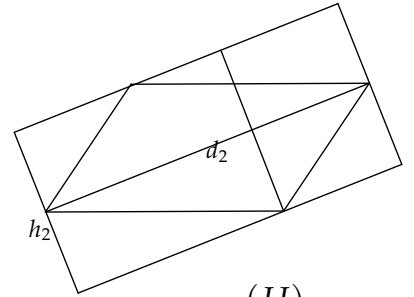

$(I I)$

Figure 2. An ellipse (I) and a parallelogram (II) that are bounded by the rectangle with length $d$ and height $h$.

In a similar way, a bound to the area is

$$
\frac{d h}{2} \leq A \leq d h .
$$


The lower bound in Equation (5) is the area of the desired quadrilateral consisting of the two triangles with the diameter of $\Omega$ as a shared base. The upper bound in Equation (5) is the area of the rectangle that contains $\Omega$.

1. Since

$$
\frac{A}{L} \leq \frac{d \cdot h}{\sqrt{d^{2}+h^{2}}}
$$

and $h<2$ for a row parallel covering, then $\frac{A}{L}<2$.

2. $\frac{h}{2 \pi}=\frac{d \cdot h / 2}{\pi \cdot d} \leq \frac{A}{L}<\frac{1}{\pi}$. Thus, the width of the bounding rectangle is less than 2 , and it admits a row cover of unit discs.

Theorem 4. Given a convex domain $\Omega$ with area $A$ and perimeter $L$ satisfying $\pi A<L$, then $\Omega$ admits a parallel row covering using at most

$$
\left[\frac{L}{4 \sqrt{1-\left(\frac{A \pi}{L}\right)^{2}}}+1\right]
$$

unit discs.

Proof. We will start by bounding $d, h$. From (4) and (5), we can obtain

$$
d \geq \frac{L}{\pi}
$$

and

$$
h \leq \frac{2 A}{d} .
$$

By substituting (6) in (7), we conclude that

$$
h \leq \frac{2 A \pi}{L} .
$$

Denote the distance between the centers of every two neighboring circles in the parallel row covering by $2 a$, with the requirement $a^{2}+\left(\frac{h}{2}\right)^{2}=1$. We also define $a=1-\varepsilon^{\prime}$ $\left(0<\varepsilon^{\prime}<1\right)$. The desired number of unit circles that cover $\Omega$ is $n:=\left\lceil\frac{d}{2 a}\right\rceil$ ( $d$ is the diameter).

Solving the equation, $1=\left(\frac{h}{2}\right)^{2}+\left(1-\varepsilon^{\prime}\right)^{2}$

leads to $h=2 \sqrt{1-\left(1-\varepsilon^{\prime}\right)^{2}}$, by inequality (8)

$$
h=2 \sqrt{1-\left(1-\varepsilon^{\prime}\right)^{2}} \leq \frac{2 A \pi}{L} .
$$

Taking the maximal height leads to

$$
\varepsilon^{\prime}=1-\sqrt{1-\left(\frac{A \pi}{L}\right)^{2}} .
$$

So, we choose

$$
a=\sqrt{1-\left(\frac{A \pi}{L}\right)^{2}} .
$$


Therefore, the desired number of unit circles is $n=\left\lceil\frac{d}{2 a}\right\rceil<\left\lceil\frac{L}{4 a}\right\rceil$, which is

$$
n \leq\left[\frac{L}{4 a}+1\right] \leq\left[\frac{L}{4 \sqrt{1-\left(\frac{A \pi}{L}\right)^{2}}}+1\right]
$$

\section{Covering a General Rectangle}

One may consider the following method of obtaining a covering of a domain, $\Omega$ : Impose $\Omega$ onto a hexagonal tiling of the plane, in which each hexagon can be inscribed by a unit circle. Then, place a unit disc at the center of each hexagon which intersects $\Omega$. This method guarantees a covering. If the lattice is as sparse as possible, the covering is expected to be efficient, although it is not guaranteed to be optimal. Finding the optimal placement of $\Omega$ onto the tiling can be done optimally using an algorithm such as the one suggested in [9]. However, to bound the cardinality of the covering from above, one may consider a random placement of $\Omega$ where the orientation is chosen to optimize the result, and the location is random and uniform.

It should be noted that the calculations presented here are for thin rectangles. However, the same method can be applied to any thin domain, and the optimal location of these domains relative to the lattice can also be deduced using the same algorithm.

We will make use of the following Theorem, which is proven in Appendix A.

Theorem 5. Given domains $K_{0}$ and $K_{1}$, where $K_{1}$ is of the form $K_{1}=K^{\prime}+(\alpha, \beta)$ (translation in the plane to $\alpha, \beta)$ ), and $(\alpha, \beta)$ is chosen uniformly in a ball with radius $r$ (where $r$ is larger than the sum of diameters of $K_{0}$ and $\left.K_{1}\right)$, then $P\left(K_{0} \cap K_{1} \neq \varnothing\right)=\frac{\operatorname{Area}\left(K_{0}+\left(-K_{1}\right)\right)}{\pi r^{2}}$ (where $-K_{1}$ is the reflection of $K_{1}$ ).

The number of unit discs that cover the rectangle is determined by the number of fundamental domains in the lattice, which intersect the rectangle. Thus, we would like to explore whether there exists a lattice, such that the number of fundamental regions intersecting the rectangle is smaller than the hexagonal regular lattice.

We will show in the case of a rectangle how to improve the hexagonal regular lattice in the sense of intersections between the lattice and the given rectangle.

Consider as the fundamental region in the desired tiling the hexagon having vertices $( \pm 1,0)$ and $( \pm \cos \beta, \pm \sin \beta)$ (See Figure 3$)$. We denote this hexagon by $\square_{\beta}$.

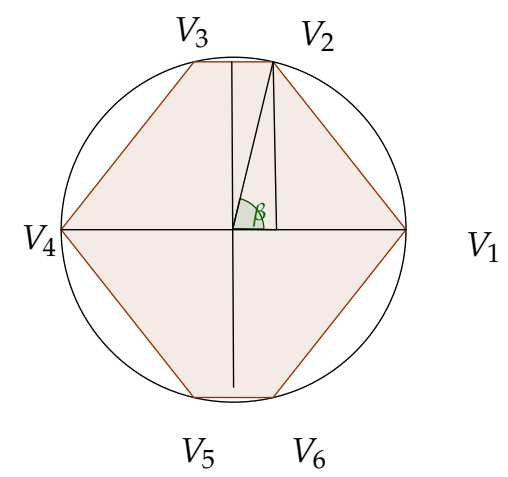

Figure 3. The desired fundamental hexagonal region, which is determined by $\beta$.

The following theorem gives us the desired tiling, which covers the rectangle. 
Theorem 6. Given a rectangle with sides $a, b$ and $b>a$. There exists $a$ tiling by hexagons as defined above with $\beta$ being the solution of

$$
4 \sin ^{4}(\beta)+8 a \sin ^{3}(\beta)+4 a^{2} \sin ^{2}(\beta)-4 a \sin (\beta)-3 a^{2}=0 \quad\left(0<\beta \leq \frac{\pi}{2}\right)
$$

such that the expected number of hexagons intersecting the rectangle is smaller than the bound given by Equation (1).

Proof. Denote the rectangle with sides $a$ and $b(b>a)$ by $\square_{(a, b)}$ and its respective area by $A\left(\square_{(a, b)}\right)$ (see Figure 4 ). By Theorem 5 , for a random placement the expected number of intersections between a given domain $K_{1}$ and a lattice is determined by the relation between the area of the Minkowski sum of the given domain and the fundamental region, in our case $\square_{(a, b)}=-\square(a, b)$, so the relation is

$$
N:=\frac{A\left(\square_{(a, b)}+\square_{\beta}\right)}{A\left(\square_{\beta}\right)},
$$

which needs to be minimized.

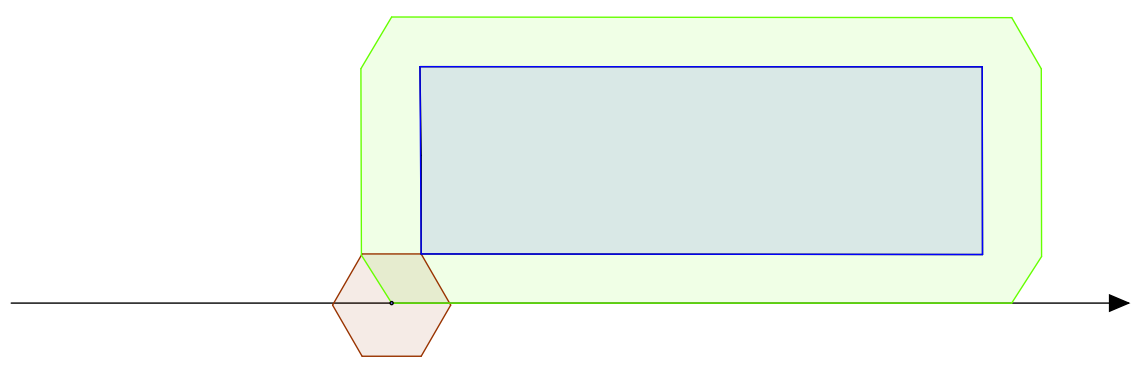

Figure 4. A Minkowski sum of a hexagon and a thin rectangle.

The Minkowsi sum $\square_{(a, b)}+\square_{\beta}$ is bounded by a rectangle with length $(b+2)$ and width $(a+2 \sin (\beta))$. So,

$$
A\left(\square_{(a, b)}+\square_{\beta}\right) \leq(a+2 \sin (\beta))(b+2) .
$$
Hence,

By Theorem 5, the number of unit discs is determined by the fundamental region.

$$
\begin{aligned}
A\left(\square_{\beta}\right) & =(4 \cdot(\sin (\beta) / 2)+2 \cdot(\sin (\beta) \cdot \sqrt{2-2 \cos (\pi-2 \beta)}) / 2 \\
& =2 \sin (\beta)+\sin (\beta) \cdot \sqrt{2+2 \cos (2 \beta)} \\
& =\sin (\beta)\left(2+\sqrt{2+2\left(2 \cos ^{2}(\beta)-1\right)}=2 \sin (\beta)(1+\cos (\beta)) .\right.
\end{aligned}
$$

So, the desired number of unit circles is at most

$$
N=f(\beta):=\frac{b+2}{2} \cdot \frac{a+2 \sin (\beta)}{\sin (\beta)(1+\cos (\beta))}
$$

Minimizing $f$ by the derivative leads to

$$
f^{\prime}(\beta)=\frac{2 \sin ^{3}(\beta)+a \sin ^{2}(\beta)-a \cos ^{2}(\beta)-a \cos (\beta)}{\sin ^{2}(\beta)(1+\cos (\beta))^{2}}=0,
$$

where $0<\beta \leq \frac{\pi}{2}$. Then,

$$
4 \sin ^{6}(\beta)+8 a \sin ^{5}(\beta)+4 a^{2} \sin ^{4}(\beta)-4 a \sin ^{3}(\beta)-3 a^{2} \sin ^{2}(\beta)=0 .
$$


Since $0<\beta \leq \frac{\pi}{2}$, then $\sin ^{2}(\beta) \neq 0$. Therefore, it is sufficient to solve the following equation:

$$
4 \sin ^{4}(\beta)+8 a \sin ^{3}(\beta)+4 a^{2} \sin ^{2}(\beta)-4 a \sin (\beta)-3 a^{2}=0,
$$

which is a quadric equation and $\beta$ can be expressed explicitly as a function of $a$.

The described optimization is for a single angle. A similar calculation can be done for general hexagonal tilings, which can be parametrized by two angels [9]. However, we conjecture that it will not improve the result, since a maximal area of the hexagon is obtained when its vertices lie on the unit circle.

Example 1. Consider a rectangle with sides $a, b$.

1. Take $a=2$ and $b \gg a$. There is a single feasible solution with $\beta=\angle V_{1} O V_{2}=50.26^{\circ}$, see Figure 5. In this case, the number of unit discs is $\left\lceil\frac{b+2}{2} \cdot 7.028\right\rceil \approx\lceil 1.403 \cdot b\rceil$, whereas the honeycomb bound given in Equation (4) gives $\left[\left(\frac{4}{3 \sqrt{3}}+\frac{4}{\pi \sqrt{3}}\right) \cdot b\right\rceil$. That is, the irregular lattice give an improvement of the bound by a factor of $\approx 1.504$.

2. Take $a=40$, then $\beta=\angle V_{1} O V_{2}=59.301^{\circ}$. Notice that since this rectangle is wide, the solution is close to the regular hexagonal lattice.

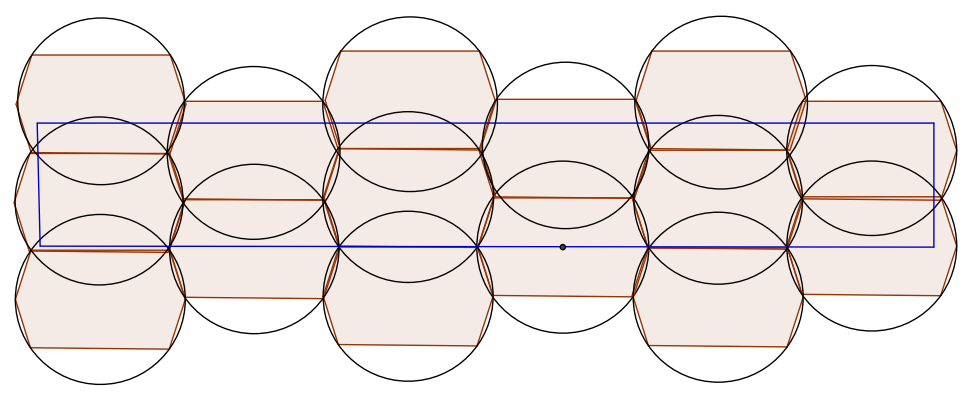

Figure 5. A covering for a rectangle by an irregular hexagonal lattice.

\section{Further Work}

Some of the results presented here can be strengthened by using stronger inequalities pertaining to convex domains. The class of hexagons discussed in Section 4 can be extended to general hexagons forming a tiling. This is a two parameter family, where the necessary condition is that each pair of opposing edges is parallel. Another possible extension is to higher dimensions. This is a challenging question, since tilings in higher dimensions are harder to characterize. Furthermore, the methods of integral geometry become more intricate in higher dimensions, as the number of parameters of the orthogonal group becomes larger and its structure becomes more complicated. However, some of the results presented here can be naturally extended to three and higher dimensions.

Author Contributions: Conceptualization, S.G. and R.C., both authors contributed equally. All authors have read and agreed to the published version of the manuscript.

Funding: This research received no external funding.

Institutional Review Board Statement: Not applicable.

Informed Consent Statement: Not applicable.

Data Availability Statement: Not applicable.

Conflicts of Interest: The authors declare no conflict of interest. 
Appendix A. The Relationship between Covering and the Minkowski Sum

Definition A1. Define the Minkowski sum

$$
T_{a b}:=\left\{(x, y) \mid(-\Omega)+\bigcirc_{a b}\right\}
$$

where $(a, b)$ is the center of the hexagon.

\section{Theorem A1.}

$$
(\alpha, \beta) \in T_{a b} \Leftrightarrow \supset_{a b} \cap E(\alpha, \beta, 0) \cdot \Omega \neq \varnothing
$$

and $E(\alpha, \beta, 0)$ shifts $\Omega$ to $(\alpha, \beta)$ (which is in the group $E(2)$ ), as in Figure A1.

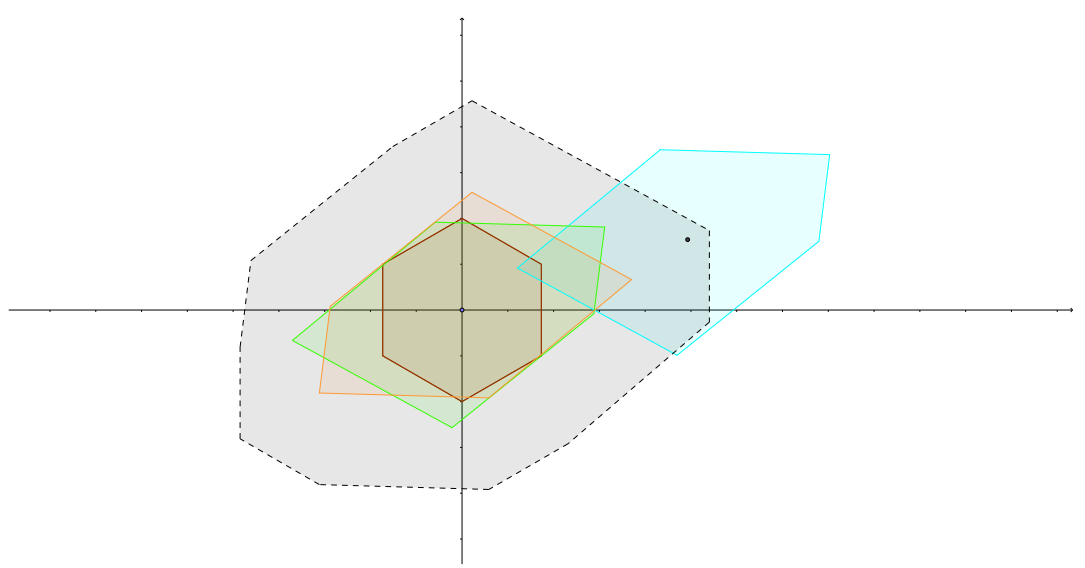

Figure A1. A hexagon at the origin intersecting a convex domain $\Omega$ (the pale blue polygon). Shifting $\Omega$ to the origin and reflecting it (the orange polygon) leads to the desired Minkowski sum $(-\Omega)+\emptyset_{0}$ (the dashed black polygon), which contains the center point of $\Omega$ (the black point).

Proof. $(\Longleftarrow)$

$$
\begin{aligned}
\bigcirc_{a b} \cap E(\alpha, \beta, 0) \cdot \Omega \neq \varnothing & \Longrightarrow \exists \bar{v}:\left(\left(\bar{v} \in \bigcirc_{a b}\right) \cap(E(\alpha, \beta, 0) \cdot \Omega)\right) \\
& \Longrightarrow \exists \bar{v}:\left(\left(\bar{v} \in \bigcirc_{a b}\right) \wedge \bar{v} \in(E(\alpha, \beta, 0) \cdot \Omega)\right) \\
& \Longrightarrow \exists \bar{v}:\left(\left(\bar{v} \in \bigcirc_{a b}\right) \wedge((\bar{v}-(\alpha, \beta)) \in \Omega)\right) \\
& \Longrightarrow \exists \bar{v}:\left(\left(\bar{v} \in \bigcirc_{a b}\right) \wedge(-(\bar{v}-(\alpha, \beta)) \in-\Omega)\right) \\
& \Longrightarrow \exists \bar{v}:\left(\left(\bar{v} \in \bigcirc_{a b}\right) \wedge(-(\bar{v}-(\alpha, \beta)) \in(-\Omega))\right) \\
& \Longrightarrow(\alpha, \beta) \in\left((-\Omega)+\bigcirc_{a b}\right) \Longrightarrow(\alpha, \beta) \in T_{a b} .
\end{aligned}
$$

$(\Longrightarrow)$

$$
(\alpha, \beta) \in T_{a b} \Longrightarrow(\alpha, \beta) \in(R(\pi) \cdot \Omega)+\bigcirc_{a b},
$$

so

$$
\begin{aligned}
\left(\left(\exists x_{1}: x_{1} \in(-\Omega)\right) \wedge\left(\exists x_{2}: x_{2} \in \bigcirc_{a b}\right)\right): x_{1}+x_{2}=(\alpha, \beta) . & \\
x_{1} \in(-\Omega) & \Longrightarrow-x_{1} \in-(-\Omega) \Longrightarrow-x_{1} \in(R(2 \pi) \cdot \Omega) \\
& \Longrightarrow-x_{1} \in \Omega \Longrightarrow\left(-x_{1}+(\alpha, \beta)\right) \in E(\alpha, \beta, 0) \cdot \Omega .
\end{aligned}
$$

Now, by (A1)

$$
x_{1}+x_{2}=(\alpha, \beta) \Longrightarrow\left(-x_{1}+(\alpha, \beta)\right)=x_{2},
$$


so, indeed $\bigcirc_{a b} \cap E(\alpha, \beta, 0) \cdot \Omega \neq \varnothing$.

Note that the above result can be obtained for every convex domain $K_{0}$ and a domain $K_{1}: E(\alpha, \beta, 0) \cdot K^{\prime}$. Since the hexagonal lattice is the optimal lattice in $\mathbb{R}^{2}$, we choose $K_{0}$ to be $\bigcirc_{a b}$.

We now prove Theorem 5 .

Proof. Denote the center of $K_{0}$ by $b_{0}$ and, respectively, $b_{1}$ for $K_{1}$. The vector $z_{0}$ is the vector, which determines $K_{0}$. In a similar way, we will define for $K_{1}$. Given $K_{0} \cap K_{1} \neq \varnothing$, there exists a point such that $b_{0}+z_{0}=b_{1}+z_{1}$, i.e., $z_{1}=z_{0}+b_{0}+\left(-b_{1}\right)$ (as in Figure A2). Without loss of generality, take $b_{0}=0$. If $b_{1} \in K_{1}$, then $-b_{1} \in\left(-K_{1}\right)$, so $z_{1} \in K_{0}+\left(-K_{1}\right)$, which is the area intersection between $K_{0}$ and $K_{1}$ in $\mathbb{R}^{2}$. So, the probability in $\mathbb{R}^{2}$ for the desired intersection is $\frac{\operatorname{Area}\left(K_{0}+\left(-K_{1}\right)\right)}{\pi r^{2}}$, where $r \gg 1$.

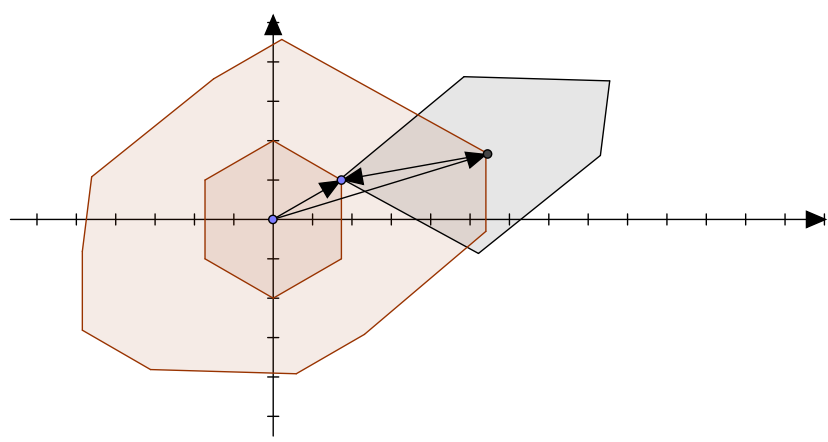

Figure A2. Constructing the support function of $K_{0}+\left(-K_{1}\right)$.

\section{References}

1. Conway, J.H.; Goodman-Strauss, C.; Sloane, N.J. Recent progress in sphere packing. Curr. Dev. Math. 1999, 1999, 37-76. [CrossRef]

2. Machchhar, J.; Elber, G. Dense packing of congruent circles in free-form non-convex containers. Comput. Aided Geom. Des. 2017, 52-53, 13-27. [CrossRef]

3. Ryu, J.; Lee, M.; Kim, D.; Kallrath, J.; Sugihara, K.; Kim, D.S. VOROPACK-D: Real-time disk packing algorithm using Voronoi diagram. Appl. Math. Comput. 2020, 375, 125076. [CrossRef]

4. $\quad$ van Sosin, B.; Rodin, D.; Sliusarenko, H.; Bartoň, M.; Elber, G. The Construction of Conforming-to-shape Truss Lattice Structures via 3D Sphere Packing. Comput.-Aided Des. 2021, 132, 102962. [CrossRef]

5. Thue, A. Über die dichteste Zusammenstellung von kongruenten Kreisen in einer Ebene. Norske Vid. Selsk. Skr. 1910, 1, 1-9.

6. Tóth, L.F. Lagerungen in der Ebene, auf der Kugel und im Raum; Springer: Berlin/Heidelberg, Germany, 1953.

7. Santaló, L. Integral Geometry and Geometric Probability; Cambridge Mathematical Library, Cambridge University Press: Cambridge, UK, 2004.

8. Qi, F.; Mahmoud, M. Some Properties of a Function Originating from Geometric Probability for Pairs of Hyperplanes Intersecting with a Convex Body. Math. Comput. Appl. 2016, 21, 27. [CrossRef]

9. Gul, S.; Cohen, R.; Haber, S. Efficient covering of convex domains by congruent discs. arXiv 2021, arXiv:2108.00707.

10. Melissen, J.; Schuur, P. Covering a rectangle with six and seven circles. Discret. Appl. Math. 2000, 99, 149-156. [CrossRef]

11. Choi, J.; Jeong, D.; Ahn, H.K. Covering Convex Polygons by Two Congruent Disks; Combinatorial Algorithms; Flocchini, P., Moura, L., Eds.; Springer International Publishing: Cham, Switzerland, 2021; pp. 165-178.

12. Basappa, M. Line segment disk cover. Discret. Appl. Math. 2021, in press. [CrossRef] 